Algebraic $8 \mathcal{G}$ Geometric $\mathcal{T}$ opology

Volume 4 (2004) 1013-1040

Published: 3 November 2004

ATG

\title{
The conjugacy problem for relatively hyperbolic groups
}

\author{
INNA BUMAGIN
}

\begin{abstract}
Solvability of the conjugacy problem for relatively hyperbolic groups was announced by Gromov [18. Using the definition of Farb of a relatively hyperbolic group in the strong sense [14, we prove this assertion. We conclude that the conjugacy problem is solvable for fundamental groups of complete, finite-volume, negatively curved manifolds, and for finitely generated fully residually free groups.
\end{abstract}

AMS Classification 20F67; 20F10

Keywords Negatively curved groups, algorithmic problems

\section{Introduction}

Relatively hyperbolic groups introduced by Gromov [18] are coarsely negatively curved relatively to certain subgroups, called parabolic subgroups. The motivating examples are fundamental groups of negatively curved manifolds with cusps that are hyperbolic relative to the fundamental groups of the cusps. Farb gave his own definition of a relatively hyperbolic group, using Cayley graphs 14, Section 3.1] (cf. Definition 2.1 below). It was first observed by Szczepanski 31. that there are groups that satisfy the Farb definition and do not satisfy the Gromov definition: $\mathbb{Z} \times \mathbb{Z}$ is an example. For this reason, groups satisfying the Farb definition are called weakly relatively hyperbolic; this terminology was suggested by Bowditch [4. Using relative hyperbolization, Szczepanski [32] obtained more examples of weakly relatively hyperbolic groups. Kapovich and Schupp [20] proved that certain Artin groups are weakly relatively hyperbolic. A weakly relatively hyperbolic group does not have to possess any nice properties. Osin 28] showed that there are weakly relatively hyperbolic groups that are not finitely presentable. He also constructed an example of a finitely presented weakly relatively hyperbolic group with unsolvable word problem.

Farb also defined and actually dealt in [14] with a somewhat restricted class of groups, namely, weakly relatively hyperbolic groups that satisfy the Bounded 
Coset Penetration (BCP) property [14, Section 3.3] (cf. Definition 2.3 below). To prove solvability of the conjugacy problem, we use this Farb's definition of relative hyperbolicity in the strong sense (see Definition 2.4 below).

Theorem 1.1 Let $G$ be a group hyperbolic relative to a subgroup $H$, in the strong sense. The conjugacy problem is solvable in $G$, provided that it is solvable in $H$.

We would like to emphasize importance of the BCP property for solvability of the conjugacy problem. Collins and Miller [10 give an example of an infinite group $G$ with a subgroup $H$ of index two (which is therefore, normal in $G$ ), so that the conjugacy problem is solvable in $H$ but is unsolvable in the whole $G$. In this example, $G$ is weakly hyperbolic relative to $H$, but normality of $H$ in $G$ violates the BCP property for the pair $(G, H)$.

Bowditch 4] elaborated the definitions given by Gromov and by Farb, and proved that Gromov's definition is equivalent to Farb's definition of relative hyperbolicity in the strong sense. A simple alternate proof of the implication "Gromov's definition $\Rightarrow$ Farb's definition in the strong sense" can be derived from the results proved in [31] and [8] (see [8] for the relevant discussion). It is worth mentioning that yet another definition of a relatively hyperbolic group was introduced by Juhasz [15].

Unlike weakly relatively hyperbolic groups, relatively hyperbolic groups in the strong sense (which we abbreviate to relatively hyperbolic groups) share many nice properties with word hyperbolic groups, provided that parabolic subgroups have similar properties. For instance, Farb proved that the word problem for a relatively hyperbolic group has "relatively fast" solution.

Theorem 1.2 14, Theorem 3.7] Suppose $G$ is strongly hyperbolic relative to a subgroup $H$, and $H$ has word problem solvable in time $O(f(n))$. Then there is an algorithm that gives an $O(f(n) \log n)$-time solution to the word problem for $G$.

Arguments that Farb used to prove this latter theorem, imply that $G$ is finitely presented, if $H$ is; moreover, $G$ has a relative Dehn presentation. Detailed proofs of these assertions, and of other basic properties of relatively hyperbolic groups were given by Osin [27. Deep results concerning boundaries and splittings of relatively hyperbolic groups were obtained by Bowditch [5], 6], [7]. Goldfarb [17] proved Novikov conjectures for these groups, and produced a 
large family of relatively hyperbolic groups using strong relative hyperbolization. Another large family of relatively hyperbolic groups was produced by Hruska [19]; these are groups acting properly discontinuously and cocompactly by isometries, on piecewise Euclidean $\mathrm{CAT}(0)$ 2-complexes with isolated flats property. A topological criterion for a group being relatively hyperbolic, was obtained by Yaman [33. Dahmani 11] proved that a relatively hyperbolic group has a finite classifying space, if its peripheral subgroups have a finite classifying space. Rebbechi 29] has shown that relatively hyperbolic groups are biautomatic, if its peripheral subgroups are biautomatic. Masur and Minsky 24] proved solvability of the conjugacy problem for mapping class groups, using the fact that a mapping class group of a surface is weakly hyperbolic relative to its subgroup that fixes a particular curve on this surface, and the pair satisfies some additional condition.

In Section [6] we apply Theorem 1.1 to prove the following.

Theorem 1.3 Let $M$ be a complete Riemannian manifold of finite volume, with pinched negative sectional curvature and with several cusps. Let $G=$ $\pi_{1}(M)$ be the fundamental group of $M$. Then there is an explicit algorithm to solve the conjugacy problem for $G$.

Dehn 13 proved that conjugacy problem for surface groups is solvable. Cannon 9] generalized Dehn's proof to all fundamental groups of closed hyperbolic manifolds. In fact, Cannon's proof works for the fundamental groups of closed negatively curved manifolds. Our result can be viewed as a generalization of Cannon's theorem to the finite volume, noncompact case.

Another example of relatively hyperbolic groups are finitely generated (f. g.) fully residually free groups which play an important role in algebraic geometry over free groups.

Definition 1.4 3] A group $L$ is fully residually free, if for any finite number $n$ of non-trivial elements $g_{1}, \ldots, g_{n}$ of $L$ there is a homomorphism $\varphi$ from $L$ into a free group $F$ so that $\varphi\left(g_{1}\right), \ldots, \varphi\left(g_{n}\right)$ are non-trivial elements of $F$.

Fully residually free groups are known to have many nice properties. For this discussion we refer the reader to deep works of Kharlampovich and Myasnikov 21], and also of Sela [30] who introduces the notion of a limit group and shows that the classes of limit groups and of f. g. fully residually free groups coincide. The following result is a conjecture of Sela, proved by Dahmani [12. Alibegovic [1] gave an alternate proof of this conjecture. 
Theorem 1.5 (Dahmani, Alibegovic) Finitely generated fully residually free groups are relatively hyperbolic with peripheral structure that consists of the set of their maximal Abelian non-cyclic subgroups.

As an immediate corollary of Theorem 1.5 and Theorem 1.1. we have solvability of the conjugacy problem for f.g. fully residually free groups.

Theorem 1.6 The conjugacy problem for finitely generated fully residually free groups is solvable.

An alternate proof of this latter assertion, based on using length functions on fully residually free groups [26], was given by Kharlampovich, Myasnikov, Remeslennikov and Serbin in the recent paper 22 .

\section{Relatively hyperbolic groups by Farb}

Definition 2.1 14] (Weakly relatively hyperbolic group) Let $G$ be a f.g. group, and let $H$ be a f.g. subgroup of $G$. Fix a set $S$ of generators of $G$. In the Cayley graph $\Gamma(G, S)$ add a vertex $v(g H)$ for each left coset $g H$ of $H$, and connect $v(g H)$ with each $x \in g H$ by an edge of length $\frac{1}{2}$. The obtained graph $\hat{\Gamma}$ is called a coned-off graph of $G$ with respect to $H$. The group $G$ is weakly hyperbolic relative to $H$ if $\hat{\Gamma}$ is a hyperbolic metric space.

The above definition depends on the choice of a generating set for $G$. Nevertheless, the property of $G$ being weakly hyperbolic relative to a subgroup $H$ is independent of this choice [14, Corollary 3.2 ]. Let $u$ be a path in $\Gamma$, we define a projection $\hat{u}$ of $u$ into $\hat{\Gamma}$ in the special case, when the generating set for $G$ contains a generating set for $H$. Reading $u$ from left to right, search for a maximal subword $z$ of generators of $H$. If $z$ goes from $g$ to $g \cdot \bar{z}$ in $\Gamma$, then we replace the path given by $z$ with the path of length 1 that goes from the vertex $g$ to the vertex $g \cdot \bar{z}$ via the cone point $v(g H)$. Do this for each maximal subword $z$ as above. In general case, projection can be defined in a similar way: we replace the path given by an element of $H$, with a path of length 1 (see 14 , Section 3.3] for details). We say that $u$ (or $\hat{u}$ ) travels $\Gamma$-distance $d_{\Gamma}(g, g \cdot \bar{z})$ in $g H$. In what follows, we assume that every path given by a maximal subword $z$ of generators of $H$, is an $H$-geodesic, in other words we always assume that $z$ is a path of the shortest $\Gamma$-length that connects $g$ and $g \cdot \bar{z}$. Having defined projection $\rho: \Gamma \longrightarrow \hat{\Gamma}$ by $\rho(u)=\hat{u}$, we can define relative (quasi)geodesics. Recall that a path $\hat{u}$ with no self-intersections in $\hat{\Gamma}$ is a $P$-quasi-geodesic if for each two 
points $x, y \in \hat{u}$ the following inequality holds: $\frac{1}{P} d_{\hat{\Gamma}}(x, y) \leq l_{\hat{u}}(x, y) \leq P d_{\hat{\Gamma}}(x, y)$ where $l_{\hat{u}}(x, y)$ denotes the length of the arc of $\hat{u}$ connecting $x$ and $y$.

Definition 2.2 14 (Relative (quasi)geodesics) If $\hat{u}$ is a geodesic in $\hat{\Gamma}$, then $u$ is called a relative geodesic in $\Gamma$. If $\hat{u}$ is a $P$-quasi-geodesic in $\hat{\Gamma}$, then $u$ is a relative $P$-quasi-geodesic in $\Gamma$.

If $\hat{u}$ passes through some cone point $v(g H)$, we say that $u$ penetrates $g H$. A path $u$ (or $\hat{u}$ ) is said to be a path without backtracking if for every coset $g H$ which $u$ penetrates, $u$ never returns to $g H$ after leaving $g H$.

Definition 2.3 14 (Bounded Coset Penetration property) Let a group $G$ be weakly hyperbolic relative to a f.g. subgroup $H$. The pair $(G, H)$ is said to satisfy the Bounded Coset Penetration (BCP) property if $\forall P \geq 1$, there is a constant $c=c(P)$ so that for every pair $u, v$ of relative $P$-quasi-geodesics without backtracking, with same endpoints, the following conditions hold:

(1) If $u$ penetrates a coset $g H$ and $v$ does not penetrate $g H$, then $u$ travels a $\Gamma$-distance of at most $c$ in $g H$.

(2) If both $u$ and $v$ penetrate a coset $g H$, then the vertices in $\Gamma$ at which $u$ and $v$ first enter (last exit) $g H$ lie a $\Gamma$-distance of at most $c$ from each other.

Definition 2.4 (Strong relative hyperbolicity) Let $G$ be a f.g. group, and let $H$ be a f.g. subgroup of $G$. We say that $G$ is hyperbolic relative to $H$ in the strong sense, if $G$ is weakly hyperbolic relative to $H$, and the pair $(G, H)$ satisfies the BCP property.

\section{Notation}

Whenever $w$ is a path in $\Gamma$, the projection of $w$ into $\hat{\Gamma}$ is denoted by $\hat{w}$. Given elements $u$ and $v$ in $G$, we assume that the equality

$$
u=g v g^{-1}
$$

holds for some $g \in G$. We denote by $w$ the closed path in $\Gamma$ labelled by $u g v^{-1} g^{-1}$. Let $w_{u}$ and $w_{v}$ be the subpaths of $w$ labelled by $u$ and $v^{-1}$, respectively. We denote by $p$ and $q$ the other two subpaths of $w$. We fix an orientation of these paths according to the equality $w=w_{u} p w_{v} q^{-1}$, so that both paths $p$ and $q$ are labelled by $g$. Due to the following lemma, we can always assume that $w_{u}, w_{v}, p$ and $q$ are relative geodesics. 
Lemma 3.1 Given an element $x \in G$, one can find effectively a relative geodesic $\gamma$ that represents $x$.

Proof We denote by $x$ both the given element and a $\Gamma$-path that represents it. Observe that $\hat{x}$ and $\hat{\gamma}$ form a pair of $l_{\Gamma}(x)$-quasi-geodesics with same endpoints. If $x$ and $\gamma$ never penetrate the same coset, then $l_{\Gamma}(\gamma) \leq l_{\Gamma}(x) c\left(l_{\Gamma}(x)\right)$. If $x$ and $\gamma$ penetrate a coset $f H$ and $x$ travels along $h_{x}$ in $f H$, then $\gamma$ travels a distance bounded by $l_{\Gamma}\left(h_{x}\right)+2 c\left(l_{\Gamma}(x)\right)$ inside $f H$. Altogether, the $\Gamma$-length of $\gamma$ is bounded as follows: $l_{\Gamma}(\gamma) \leq l_{\Gamma}(x)\left(2 c\left(l_{\Gamma}(x)\right)+1\right)$. There are only finitely many elements of $G$ whose length is bounded as above. Find those that are equal to $x$ in $G$ and take one whose relative length is minimal possible.

Corollary 3.2 Given an element $u \in G$, one can determine effectively whether or not $u$ is in $H$.

For a fixed set $S$ of generators of $G$, let $S^{ \pm 1}$ denote the set of those generators and their inverses. A product $g_{i_{1}} g_{i_{2}} \ldots g_{i_{k}}$ of elements of $S^{ \pm 1}$ is a reduced word, if $g_{i_{j+1}} \neq g_{i_{j}}^{-1}$ for all $j=1,2, \ldots, k-1$. We assume that relative geodesics are labelled by reduced words. A reduced word $g_{i_{1}} g_{i_{2}} \ldots g_{i_{k}}$ is cyclically reduced, if $g_{i_{1}} \neq g_{i_{k}}^{-1}$. We say that an element $x \in G$ is cyclically reduced, if the label of each relative geodesic $\gamma_{x}$ that represents $x$ (see Lemma 3.1), is a cyclically reduced word. Observe that if $x$ is not cyclically reduced, then a relative geodesic $\gamma_{x}$ has a proper subpath $\gamma_{y}$ which is labelled by a cyclically reduced word. $\gamma_{y}$ represents an element $y$ of $G$ which is conjugate to $x$. Since there are only finitely many candidates for $\gamma_{x}$ and hence for $\gamma_{y}$, we can assume that $\gamma_{y}$ has the minimal possible relative length. For the conjugacy problem, we can work with $y$ instead of $x$. If $\gamma_{y}$ is a relative geodesic, then we are done. Otherwise, we will proceed with elements of shorter relative length; therefore, the process will eventually stop. In what follows, we assume that $u$ and $v$ are cyclically reduced elements of $G$.

Let $Q=\max \left\{l_{\Gamma}(u), l_{\Gamma}(v)\right\}$ denote the maximal length of $u$ and $v$, and let $\hat{Q}=$ $\max \left\{l_{\hat{\Gamma}}(\hat{u}), l_{\hat{\Gamma}}(\hat{v})\right\}$ denote the maximal relative length of $\hat{u}$ and $\hat{v}$. Let $L=l_{\hat{\Gamma}}(\hat{w})$ be the relative length of $w$, and let $C=c(L)$ be the constant introduced in Definition 2.3. Observe that the closed path $\hat{w}$ is the concatenation of two $L$-quasi-geodesic paths as follows: $\lambda_{1}=w_{u} p$ and $\lambda_{2}=w_{v} q^{-1}$. Denote by $l_{H}^{u}$ (or $l_{H}^{v}$ ) the maximal distance which $u$ (or $v$ ) travels in an $H$-coset. 


\section{Conjugacy problem for hyperbolic groups}

In this section we show that the conjugacy problem for hyperbolic groups is solvable (see also [18, 23]). Our proof for relatively hyperbolic groups is based in part on the extension of similar ideas to a more general situation, and uses some of the results proven in this section. To show solvability of the conjugacy problem for hyperbolic groups, we study properties of quasi-geodesics in a hyperbolic space. Observe that if $G$ is a hyperbolic group, then $G$ is hyperbolic relative to the trivial subgroup so that the coned-off graph $\hat{\Gamma}$ and the Cayley graph $\Gamma$ of $G$ coincide.

Lemma 4.1 (Concatenation of two paths in a geodesic space) Let $\alpha=\alpha_{1} \cdot \alpha_{2}$ be the concatenation of a geodesic $\alpha_{1}$ and of a non-empty path $\alpha_{2}$ in a geodesic metric space $\Delta$, so that $\alpha$ does not intersect itself and $l_{\Delta}\left(\alpha_{1}\right) \geq 2 l_{\Delta}\left(\alpha_{2}\right)$. Then $\alpha$ is a $\left(2 l_{\Delta}\left(\alpha_{2}\right)+1\right)$-quasi-geodesic.

Proof Let $\beta$ be a geodesic with the same endpoints as $\alpha$. Then $l_{\Delta}\left(\alpha_{2}\right) \leq$ $\frac{1}{2} l_{\Delta}\left(\alpha_{1}\right) \leq l_{\Delta}(\beta)$. Hence, $\frac{l_{\Delta}\left(\alpha_{1}\right)+l_{\Delta}\left(\alpha_{2}\right)}{l_{\Delta}(\beta)} \leq 3$. Now, let $x_{i} \in \alpha_{i}$ be a point, let $\tilde{\alpha}$ be the subpath of $\alpha$ between $x_{1}$ and $x_{2}$, and let $\beta$ be a geodesic joining $x_{1}$ and $x_{2}$. Since $\alpha$ does not intersect itself, $l_{\Delta}(\beta) \geq 1$. It can be readily seen that the maximum possible value of the ratio $\frac{l_{\Delta}(\tilde{\alpha})}{l_{\Delta}(\beta)}$ equals $2 l_{\Delta}\left(\alpha_{2}\right)+1$. Also, note that $2 l_{\Delta}\left(\alpha_{2}\right)+1 \geq 3$.

Lemma 4.2 If $l_{\hat{\Gamma}}(\hat{g}) \geq 3 \hat{Q}$, then the closed path $\hat{w}$ is the concatenation of two $(2 \hat{Q}+1)$-quasi-geodesics. Moreover, without loss of generality one can assume that the paths $\lambda_{1}=\hat{w}_{u} \hat{p}$ and $\lambda_{2}=\hat{q} \hat{w}_{v}^{-1}$ are $(2 \hat{Q}+1)$-quasi-geodesics.

Proof We prove the assertion for $\lambda_{1}$, the proof for $\lambda_{2}$ is similar. We only need to show that $\lambda_{1}$ has no self-intersection. Assume, $\lambda_{1}$ intersects itself, which means that $\hat{w}_{u}$ and $\hat{p}$ have at least two points in common. Let $x$ be the point were $\hat{w}_{u}^{-1}$ and $\hat{p}$ last intersect: $x=\hat{p}(t)=\hat{w}_{u}^{-1}(t)$, for some $t$. The path $\hat{w}$ will remain closed if we choose $g$ so that $\hat{p}$ coincides with $\hat{w}_{u}^{-1}$ till $x$. Since $u$ is cyclically reduced, $\hat{w}_{u}$ and $\hat{q}$ do not intersect. Therefore, the path $\tilde{\lambda}$ that starts at $\hat{q}(t)$, goes through $\hat{w}_{u}$ till $x$ and then through the rest of $\hat{p}$, satisfies the conditions of Lemma 4.1 so that the first assertion of the lemma follows.

To prove the second assertion, note that the initial segment $\left[\hat{q}(t), \hat{w}_{u}^{-1}(t)\right]$ of $\tilde{\lambda}$ represents a cyclic conjugate $\tilde{u}$ of $u$. Also note that in the closed path $\tilde{w}$ formed by "cutting off" the common segment of $\hat{w}_{u}$ and $\hat{p}$, the path $\tilde{\lambda}$ plays the role of $\lambda_{1}$. 
Corollary 4.3 Let $l_{\hat{\Gamma}}(\hat{g}) \geq 3 \hat{Q}$. If $\lambda_{1}=\hat{w}_{u} \hat{p}$ backtracks, so that it can be shortened, then this shorter path $\tilde{\lambda}_{1}$ is a $(2 \hat{Q}+1)$-quasi-geodesic.

Proof $\tilde{\lambda}_{1}=\alpha_{1} \cdot \alpha_{2}$ is the concatenation of a path $\alpha_{1}$ with $l_{\hat{\Gamma}}\left(\alpha_{1}\right) \leq \hat{Q}$ and of a geodesic $\alpha_{2}$ with $l_{\hat{\Gamma}}\left(\alpha_{2}\right) \geq 2 \hat{Q}$. The assertion follows from Lemma 4.1

Observe that the proofs of Lemma 4.1. Lemma 4.2 and Corollary 4.3 do not use the assumption that $\hat{\Gamma}$ is hyperbolic, so that these statements hold for any geodesic metric space. However, we cannot drop the assumption that $\hat{\Gamma}$ is hyperbolic, in Corollary 4.4 and Lemma 4.5 below.

Corollary 4.4 The paths $\lambda_{1}=\hat{w}_{u} \hat{p}$ and $\lambda_{2}=\hat{q} \hat{w}_{v}^{-1}$ stay a bounded distance $K$ from each other in $\hat{\Gamma}$; moreover, $K$ does not depend on the $\hat{\Gamma}$-length of $\hat{p}$ (or $\hat{q})$.

Proof If $l_{\hat{\Gamma}}(\hat{g}) \leq 3 \hat{Q}$, then the relative length of $\lambda_{1}$ and of $\lambda_{2}$ is bounded by $4 \hat{Q}$, so that $\lambda_{1}$ and $\lambda_{2}$ stay a distance bounded by $2 \hat{Q}$ from each other. Otherwise, by Lemma4.2, $\lambda_{1}$ and $\lambda_{2}$ are $(2 \hat{Q}+1)$-quasi-geodesics with common endpoints. Therefore, they stay a bounded distance $N(2 \hat{Q}+1)$ from each other. In order to obtain the claim, set

$$
K=\max \{2 \hat{Q}, N(2 \hat{Q}+1)\} .
$$

Note that the initial point of both $\hat{w}_{u}$ and $\hat{q}$ is the identity $1_{\hat{\Gamma}}$, and that the initial point of $\hat{p}$ coincides with the terminal point of $\hat{w}_{u}$.

Lemma 4.5 Start at the initial points of $\hat{p}$ and $\hat{q}$ and move along these paths with the unit speed. If $\hat{p}$ and $\hat{q}$ are long enough paths, then there exist two numbers $t_{1}, t_{2}$ satisfying $1 \leq t_{1}<t_{2} \leq l_{\hat{\Gamma}}(\hat{p})$, so that the following condition holds. For each integer $t$ where $t_{1} \leq t \leq t_{2}$, the $\hat{\Gamma}$-length of a geodesic path $\hat{\gamma}$ joining $\hat{p}(t)$ and $\hat{q}(t)$ can be bounded in terms of the $\hat{\Gamma}$-lengths of $u$ and $v$.

Proof Assume that $\hat{p}$ and $\hat{q}$ are longer than $3 \hat{Q}+2 \delta$ so that we can consider values of $t_{1}, t_{2}$ as follows:

$$
l_{\hat{\Gamma}}(\hat{u})+\delta=t_{1}<t_{2}=l_{\hat{\Gamma}}(\hat{p})-\left(l_{\hat{\Gamma}}(\hat{v})+\delta\right) .
$$

Denote by $\hat{p}^{\prime}$ (or $\hat{q}^{\prime}$ ) the subpath of $\hat{p}$ (or $\hat{q}$ ) between $\hat{p}\left(t_{1}\right)$ (or $\hat{q}\left(t_{1}\right)$ ) and $\hat{p}\left(t_{2}\right)$ (or $\left.\hat{q}\left(t_{2}\right)\right)$. Because of our choice of $t_{1}$ and $t_{2}$, the geodesics $\hat{p}^{\prime}$ and $\hat{q}^{\prime}$ stay the distance $K$ from each other ( $K$ as in (2) ). Furthermore, the distance 
between the initial points of the paths $\hat{p}^{\prime}$ and $\hat{q}^{\prime}$ is bounded above as follows: $d_{\hat{\Gamma}}\left(\hat{p}\left(t_{1}\right), \hat{q}\left(t_{1}\right)\right) \leq l_{0}$, where $l_{0}=3 \hat{Q}+2 \delta$.

Note that $d_{\hat{\Gamma}}\left(\hat{p}\left(t_{1}\right), \hat{p}(t)\right)=d_{\hat{\Gamma}}\left(\hat{q}\left(t_{1}\right), \hat{q}(t)\right)=\left|t-t_{1}\right|$. Consider $\hat{\gamma}(t)$ for $t_{1}<t<$ $t_{2}$. There is a point $x=\hat{q}\left(t_{x}\right)$ so that $d_{\hat{\Gamma}}(\hat{p}(t), x) \leq K$. Assume, $t_{1} \leq t_{x} \leq t$, so that $d_{\hat{\Gamma}}(\hat{q}(t), x)=\left|t-t_{1}\right|-d_{\hat{\Gamma}}\left(\hat{q}\left(t_{1}\right), x\right)$. Since $\hat{p^{\prime}}$ is a geodesic, we have that $\left|t-t_{1}\right| \leq l_{0}+d_{\hat{\Gamma}}\left(\hat{q}\left(t_{1}\right), x\right)+K$. Hence, $d_{\hat{\Gamma}}(\hat{q}(t), x) \leq l_{0}+K$. If $t_{x} \geq t$, then we have that $d_{\hat{\Gamma}}\left(\hat{q}\left(t_{1}\right), x\right)=\left|t-t_{1}\right|+d_{\hat{\Gamma}}(\hat{q}(t), x) \leq K+\left|t-t_{1}\right|+l_{0}$, so that $d_{\hat{\Gamma}}(\hat{q}(t), x) \leq l_{0}+K$ as well. In both cases, we conclude that

$$
l_{\hat{\Gamma}}(\hat{\gamma}(t))=d_{\hat{\Gamma}}(\hat{p}(t), \hat{q}(t)) \leq 3 \hat{Q}+2 \delta+2 K,
$$

which implies the claim.

As a consequence, we get the following theorem which is the main result of this section.

Theorem 4.6 If $G$ is a hyperbolic group, then the conjugacy problem in $G$ is solvable.

Proof It follows immediately from Lemma 4.5 that for $\hat{p}$ and $\hat{q}$ long enough, one can find two integers $s_{1}$ and $s_{2}$ satisfying the double inequality $t_{1} \leq s_{1}<$ $s_{2} \leq t_{2}$, so that two geodesics $\hat{\gamma}_{i}$ connecting $\hat{p}\left(s_{i}\right)$ with $\hat{q}\left(s_{i}\right)(i=1,2)$ have the same $\hat{\Gamma}$-length. Therefore, the compactness of balls of a given radius in $\hat{\Gamma}$ implies that for $\hat{p}$ and $\hat{q}$ long enough, we can find $\hat{\gamma}_{1}, \hat{\gamma}_{2}$ as above so that these geodesics represent the same element $x$ of $G$. In this case, both $u$ and $v$ are conjugate to $x$ in $G$. Moreover, we can cut off the segments $\left[\hat{p}\left(s_{1}\right), \hat{p}\left(s_{2}\right)\right]$ and $\left[\hat{q}\left(s_{1}\right), \hat{q}\left(s_{2}\right)\right]$ of $\hat{p}$ and $\hat{q}$, respectively, and obtain a shorter element conjugating $u$ and $v$. Thus, if two elements $u$ and $v$ of a hyperbolic group are conjugate, then the minimal possible length of a conjugating element $g$ is bounded in terms of the lengths of $u$ and $v$ (cf. [23, Lemma 10]). Since bounded balls in $\hat{\Gamma}$ are compact and word problem in $G$ is solvable, the assertion follows.

\section{$5 \quad$ Relatively hyperbolic groups}

We assume that the conjugacy problem is solvable in $H$. Therefore, given $u, v \in$ $H$, we can determine effectively whether or not $u$ and $v$ are conjugate in $H$. If this is the case, then $\hat{w}$ is the null-path in $\hat{\Gamma}$. In what follows, we assume that $L=l_{\hat{\Gamma}}(\hat{w})>1$. 
Definition 5.1 (Closed path without backtracking) We will say that a closed path $\hat{w}$ does not backtrack to a coset $g H$ which it penetrates, if $\hat{w}$ is the concatenation of two paths $\hat{u}$ and $\hat{v}^{-1}$ so that $\hat{u}$ is a path without backtracking which penetrates $g H$, and $\hat{v}$ does not penetrate $g H$. We will say that the closed path $\hat{w}$ is a path without backtracking, if for any coset $g H$ which it penetrates, $\hat{w}$ does not backtrack to $g H$.

The following results which will be used later on, are straightforward consequences of the Definition 2.3 of the BCP property. Recall that $C=c(L)$ is the constant introduced in Definition 2.3.

Lemma 5.2 If $\hat{w}$ penetrates a coset $g H$ and does not backtrack to it, then $\hat{w}$ travels in $g H$ a $\Gamma$-distance of $C=c(L)$ at most.

Proof Since each relative $P$-quasigeodesic is in particular an $R$-quasigeodesic for $P<R$, we have that $c(P) \leq c(R)$. Let $\hat{w}$ travel in $g H$ along an $H$ geodesic $h$. Consider the subpath $\hat{w}_{1}=g_{1} h g_{2}$ of $\hat{w}$. The relative length of $\hat{w}_{1}$ equals 3 so that $\hat{w}_{1}$ is a 3 -quasigeodesic. If $\hat{w}_{1}$ is a closed path, then we have found a pair of two relative 2-quasigeodesics, one of which penetrates the coset $g H$, and the other does not penetrate $g H$. Therefore, in this case $L_{\Gamma}(h) \leq c(2) \leq c(L)$. Now, assume $\hat{w}_{1}$ is not a closed path. Let $\hat{w}_{2}$ be so that $\hat{w}=\hat{w}_{1} \hat{w}_{2}$. Since $\hat{w}$ does not backtrack to $g H$, $\hat{w}_{2}$ does not penetrate this coset. If $\hat{w}_{2}$ backtracks to a coset different from $g H$, then we can shorten $\hat{w}_{2}$ each time when backtracking occurs. Indeed, assume $\hat{w}_{2}$ leaves a coset $f H$ at some point $x$ and enters this coset at $y$, later on. We replace the subpath of $\hat{w}_{2}$ joining $x$ and $y$, with an $H$-geodesic $h_{x, y}$ joining these points. Finally, we obtain a path $\hat{w}_{2}^{\prime}$ without backtracking, with $L_{\hat{\Gamma}}\left(\hat{w}_{2}^{\prime}\right) \leq L-3$. Hence, $\hat{w}_{2}^{\prime}$ is a relative $(L-3)$-quasigeodesic, which does not penetrate $g H$. Therefore, the pair $\hat{w}_{1}$ and $\hat{w}_{2}^{\prime}$ of relative $(L-3)$-quasigeodesics satisfies the first part of Definition 2.3] and it follows that $L_{\Gamma}(h) \leq c(L-3) \leq c(L)$.

Lemma 5.3 Assume that $\hat{w}_{u}$ and $\hat{p}$ penetrate a coset $f H$, but neither $\hat{q}$ nor $\hat{w}_{v}$ penetrates $f H$. Let $k_{1}$ (or $k_{2}$ ) be a $\Gamma$-geodesic joining the points where $\hat{w}_{u}^{-1}$ and $\hat{p}$ first enter (or last exit) the coset $f H$. Then $l_{\Gamma}\left(k_{1}\right) \leq c(2)$ and $l_{\Gamma}\left(k_{2}\right) \leq C$ (Figure 1).

Proof Denote by $x_{p}$ and $x_{u}$ (or $y_{p}$ and $y_{u}$ ) the endpoints of $k_{1}$ (or of $k_{2}$ ). To prove the inequality for $l_{\Gamma}\left(k_{1}\right)$, consider the following pair of 2-quasi-geodesics: $\alpha$ is the concatenation of the initial subgeodesic of $\hat{p}$ (ending at $x_{p}$ ) and $k_{1}, \beta$ is 


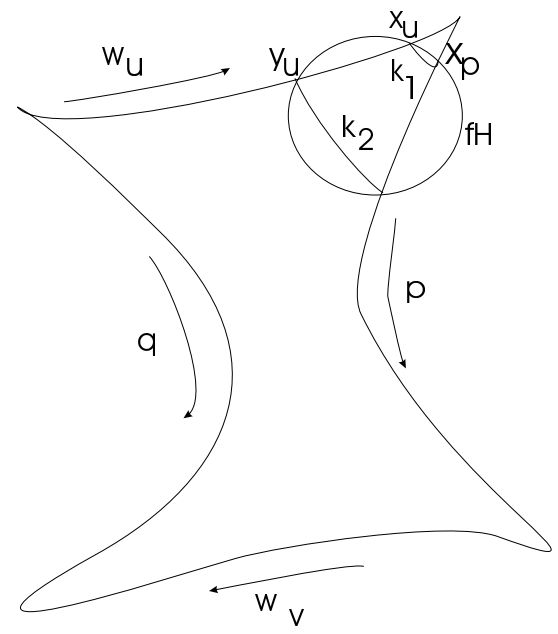

Figure 1: Illustrated above is the case when $\hat{w}_{u}$ and $\hat{p}$ penetrate a coset $f H$, which $\hat{q}$ and $\hat{w}_{v}$ do not penetrate (Lemma [5.3).

the initial subgeodesic of $\hat{w}_{u}^{-1}$ (ending at $x_{u}$ ). Note that $\alpha$ and $\beta$ have common endpoints and do not backtrack. Therefore, the first part of the definition of the $\mathrm{BCP}$ property implies the claim for $k_{1}$. To prove the inequality for $l_{\Gamma}\left(k_{2}\right)$, consider the closed path $\hat{w}^{\prime}$ which is the concatenation of the following geodesics: $k_{2}$, the terminal subgeodesic of $\hat{w}_{u}^{-1}$ (starting at $y_{u}$ ), $\hat{q}, \hat{w}_{v}^{-1}$ and the initial subgeodesic of $\hat{p}^{-1}$ (ending at $y_{q}$ ). As $\hat{w}^{\prime}$ is shorter than $\hat{w}$, Lemma 5.2 implies the claim for $k_{2}$.

Corollary 5.4 Let $\lambda_{1}=w_{u} p$ and $\lambda_{2}=w_{v} q^{-1}$ be relative $P$-quasi-geodesics for some $P>0$. Let $p$ travel along the path $h_{g}$ in a coset $f H$. If $p$ and $q$ do not penetrate the same coset, then $l_{\Gamma}\left(h_{g}\right) \leq 2 Q+c(P)+2 c(2)$.

Proof Let $\hat{w}_{u}$ and $\hat{p}$ penetrate a coset $f H$. The proof of Lemma 5.3 works verbatim in the case when $\hat{w}_{v}$ does not penetrate the coset $f H$, but instead of Lemma 5.2 use Corollary 4.3 Now, assume $\hat{w}_{v}$ penetrates $f H$ also. Let $k_{1}$ be as in the statement of Lemma 5.3. let $k_{2}$ be a geodesic joining the points at which $\hat{w}_{v}$ and $\hat{p}^{-1}$ first enter $f H$, and let $k_{3}$ join the points at which $\hat{w}_{u}$ and $\hat{w}_{v}^{-1}$ first enter $f H$. It follows from the argument used to prove Lemma 5.3 that $l_{\Gamma}\left(k_{i}\right) \leq c(2)$ for $i=1,2$. Moreover, it can be readily seen that the concatenation of the initial segment of $\hat{w}_{u}$ and of $k_{3}$ is a relative 3-quasigeodesic. By assumption, the concatenation of $\hat{q}^{-1}$ and of the initial segment of $w_{v}^{-1}$ is a relative $P$-quasi-geodesic which does not penetrate $f H$, so that $l_{\Gamma}\left(k_{3}\right) \leq c(P)$, which implies the claim. 
Remark. Corollary 5.4 holds for each closed path which is the concatenation of four geodesics. Indeed, the fact that $\hat{p}$ and $\hat{q}$ have the same label is never used in the proof of this statement.
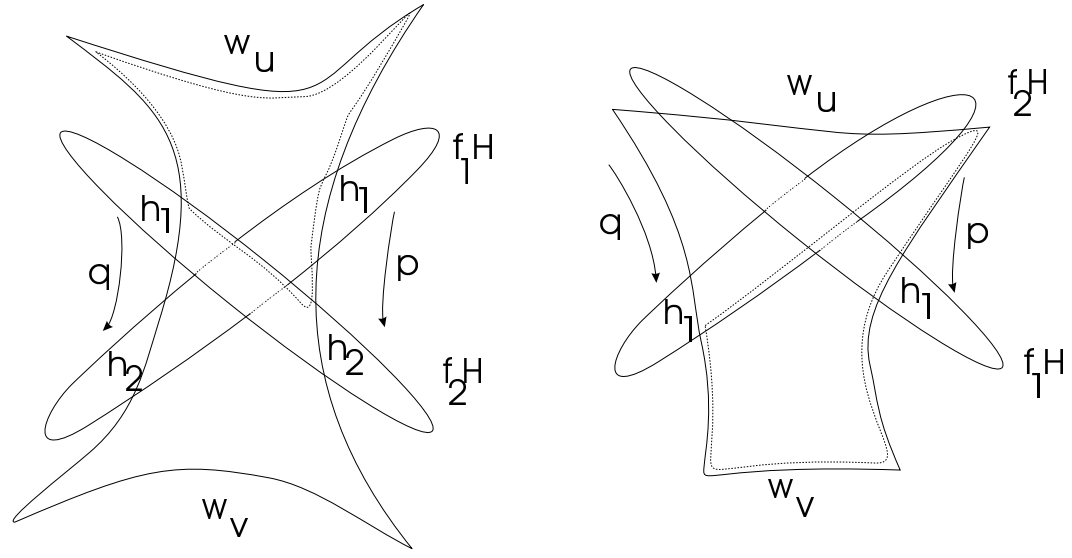

Figure 2: Examples of "skew" cosets (Lemma [5.5). The dotted line shows a closed path which does not backtrack and travels a bounded distance in $f_{1} H$.

Lemma 5.5 Assume that $\hat{p}$ and $\hat{q}$ penetrate two "skew" cosets $f_{1} H$ and $f_{2} H$ so that $\hat{p}$ travels in $f_{1} H$ along $h_{1}$ and $\hat{q}$ travels in $f_{2} H$ along $h_{1}$, and either

(1) $\hat{w}_{u}$ and $\hat{w}_{v}$ penetrate neither of these cosets, and $\hat{p}$ travels in $f_{2} H$ along $h_{2}$ while $\hat{q}$ travels in $f_{1} H$ along $h_{2}$ (Figure 2] left), or

(2) $\hat{w}_{u}$ penetrates first $f_{1} H$ and then $f_{2} H, \hat{w}_{v}$ penetrates neither of these cosets, $\hat{p}$ does not penetrate $f_{2} H$, and $\hat{q}$ does not penetrate $f_{1} H$ (Figure 2, right).

Then $l_{\Gamma}\left(h_{1}\right) \leq C$.

Proof We prove the assertion only in the case (1), the other case is similar. Consider the closed path $\hat{w}^{\prime}=\hat{p}_{1} \circ k \circ \hat{q}_{1}^{-1} \circ \hat{w}_{u}$, where $k$ is an $H$-geodesic in $f_{2} H$, and $\hat{p}_{1}$ (or $\hat{q}_{1}$ ) is the initial segment of $\hat{p}$ (or $\hat{q}$ ) that terminates at the point where $\hat{p}$ (or $\hat{q}$ ) first enters the coset $f_{2} H$. This path satisfies the conditions of Lemma 5.2 and is shorter than $\hat{w}$; the assertion (11) follows.

\subsection{Cascades}

So far, we were able to apply directly the definition of the BCP property in order to bound the $\Gamma$-length of a subpath of $w$ in terms of the $\hat{\Gamma}$-length of $\hat{w}$. 
It is possible unless $\hat{p}$ and $\hat{q}$ penetrate the same cosets. Contract each vertex of $\hat{\Gamma}$ to a point; if $\hat{p}$ and $\hat{q}$ penetrate the same cosets, then $\hat{w}$ will turn into a sequence of digons with two triangles at both ends of it. In the case when these triangles are isosceles, the length of the conjugating element $g$ cannot be bounded in general. We consider this case in Section 5.4 below. If the triangles are not isosceles, then we have cascade effect defined as follows.

Definition 5.6 (Cascade effect) We say that in the path $w=w_{u} p w_{v} q^{-1}$ cascade effect occurs if the following condition holds. There are subwords $h_{1}, h_{2}, \ldots, h_{n+1} \in H$ of $g$ and cosets $f_{1} H, f_{2} H, \ldots, f_{n} H$, which both $p$ and $q$ penetrate in the same order, and so that either $p$ travels in the coset $f_{i} H$ along $h_{i}$ and $q$ travels in the coset $f_{i} H$ along $h_{i+1}$ (Figure 3, left), or $p$ travels in the coset $f_{i} H$ along $h_{i+1}$ and $q$ travels in the coset $f_{i} H$ along $h_{i}$, for each $i=1,2, \ldots, n$. The number $n$ is called the length of the cascade.
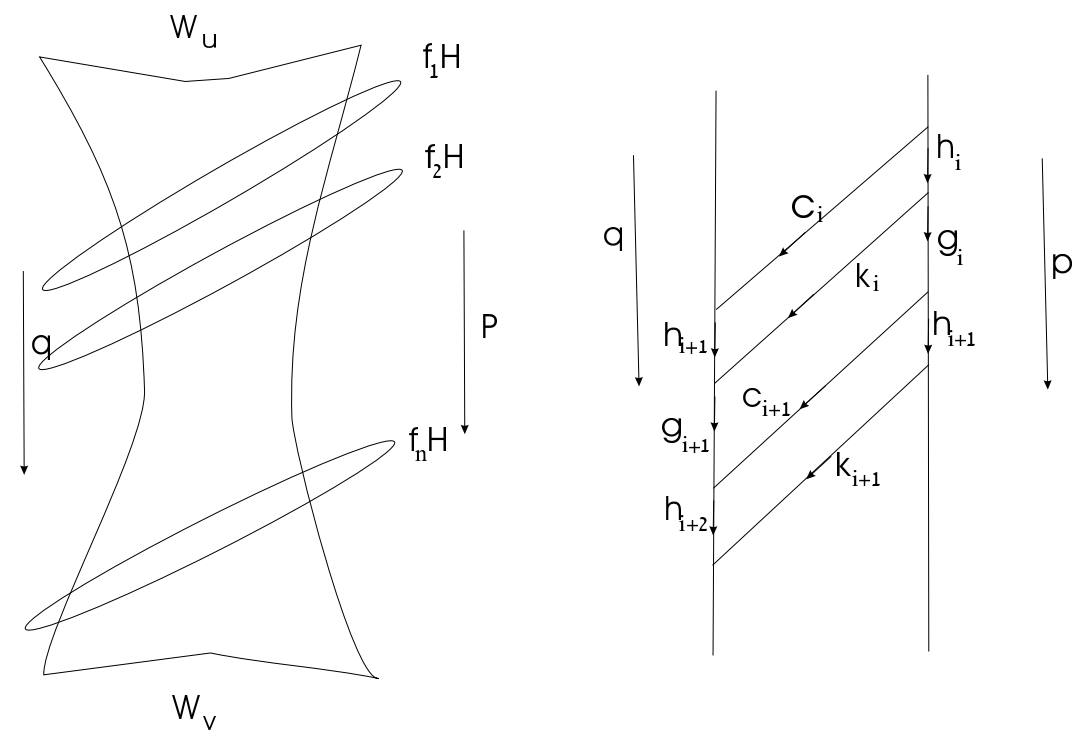

Figure 3: Left: Cascade effect. Right: Three consecutive floors of a cascade, two $H$-floors and a $G$-floor between them.

The "tower" corresponding to a cascade is an alternating sequence of $H$-floors and $G$-floors (Figure 3, right); each $H$-floor is a closed path $h_{i} k_{i} h_{i+1}^{-1} c_{i}^{-1}$ in $H$, each $G$-floor is a closed path $g_{i} c_{i+1} g_{i+1}^{-1} k_{i}^{-1}$ where $g_{i} \in G \backslash H$ and $k_{i}, c_{i} \in H$. Each $G$-floor (or $H$-floor) has two neighboring $H$-floors (or $G$-floors) glued to it along $\Gamma$-geodesic arcs corresponding to $k_{i}$ and $c_{i+1}$ (or $c_{i}$ and $k_{i}$ ). Observe that in a cascade of length $n$, the number of $H$-floors is $n$ and the number of 
$G$-floors is $n-1$. It can be readily seen that the length of the subwords $h_{i}$ of $g$ can be bounded in terms of the length of the cascade as follows: $l_{\Gamma}\left(h_{i}\right) \leq$ $l_{\Gamma}\left(h_{1}\right)+2 i c(2) \leq l_{\Gamma}\left(h_{1}\right)+2 n c(2)$ (see the part (11) of Lemma 5.7 below); the main difficulty is to handle cases when $n$ is large. In Lemma 5.8 below we obtain a bound on the length of $h_{i}$ which does not depend on the length of a cascade. Lemma 5.7 asserts that the $\Gamma$-length of each $k_{i}$ and of each $c_{i}$ can be bounded in terms of the relative length of $u$ and $v$. Since we can skip several $H$-floors in the beginning and in the end of a cascade, we will always assume that neither $w_{u}$ nor $w_{v}$ penetrate the cosets where $H$-floors of a cascade are located. We set $C_{0}=c(7 Q)$.

Lemma 5.7 With the notation above, the length of the $\Gamma$-geodesics $c_{i}$ and $k_{i}$ can be bounded as follows.

(1) $l_{\Gamma}\left(k_{i}\right) \leq c(2)$ for $1 \leq i<n$, and $l_{\Gamma}\left(c_{i}\right) \leq c(2)$ for $1<i \leq n$.

(2) $l_{\Gamma}\left(k_{n}\right) \leq C_{0}$ and $l_{\Gamma}\left(c_{1}\right) \leq C_{0}$.

Proof As each $G$-floor is the concatenation of two 2-geodesics, the assertion (11) follows immediately. We prove the assertion (2) for $c_{1}$, the proof for $k_{n}$ is similar. Consider the closed path $w^{\prime}$ which is the concatenation of $w_{u}, p_{1}$, $c_{1}$ and $q_{1}^{-1}$, where $p_{1}$ and $q_{1}$ are the initial segments of $p$ and $q$, respectively. Without loss of generality, assume that $l_{\Gamma}\left(p_{1}\right) \leq l_{\Gamma}\left(q_{1}\right)$. The concatenation $\alpha=p_{1} \cdot c_{1}$ is a relative 2 -quasi-geodesic.

If $p_{1}$ and $q_{1}$ penetrate a coset $f H$, then a subpath of $\alpha$ as well as the concatenation of a $\Gamma$-geodesic which travels in $f H$ and of $q_{1}^{\prime}$ are relative 2-quasi-geodesics with common endpoints. Hence, $l_{\Gamma}\left(c_{1}\right) \leq c(2)$ in this case. In what follows, we assume that $p_{1}$ and $q_{1}$ do not penetrate the same coset.

We distinguish the two cases as follows:

(1) $\hat{q}_{1}$ is "long": $l_{\hat{\Gamma}}\left(\hat{q}_{1}\right) \geq 3 Q>2 l_{\hat{\Gamma}}\left(\hat{w}_{u}\right)$.

(2) $\hat{q}_{1}$ is "short": $l_{\hat{\Gamma}}\left(\hat{q}_{1}\right)<3 Q$.

In the case (11) our argument below shows that $l_{\Gamma}\left(c_{1}\right) \leq c(2 Q+1)$. Indeed, the concatenation $\hat{\beta}=\hat{w}_{u}^{-1} \cdot \hat{q}_{1}$ is a $(2 Q+1)$-quasi-geodesic, according to Lemma4.1. If $\hat{\beta}$ backtracks, then by Corollary 4.3, a shorter path $\tilde{\beta}$ without backtracking and same endpoints as $\hat{\beta}$, is a $(2 Q+1)$-quasi-geodesic. Furthermore, if $\hat{p}_{1}$ and $\hat{w}_{u}^{-1}$ penetrate a coset $f H$, then set $\tilde{\alpha}$ to be the subpath of $\hat{\alpha}$ which begins at the point where $\hat{p}_{1}$ exits $f H$, and adjust $\tilde{\beta}$ accordingly. In any case, we have a pair of $(2 Q+1)$-quasi-geodesics that satisfies the first part of the definition of the BCP property, which implies the claim in the case when $\hat{q}_{1}$ is "long". 
In the case (21) $l_{\hat{\Gamma}}\left(\hat{w}^{\prime}\right) \leq 7 Q$, and so by Lemma 5.2, $l_{\Gamma}\left(c_{1}\right) \leq C_{0}=c(7 Q)$, as claimed.

Lemma 5.8 Assume that in the word $w$ the cascade effect occurs. Let $p$ travel in the coset $f_{i} H$ along $h_{i}$. Then $l_{\Gamma}\left(h_{i}\right) \leq l_{\Gamma}\left(h_{1}\right)+2 C_{0}+2 c(2)$, for $i=2,3, \ldots, n-1$.

Proof Since each $G$-floor is the concatenation of two 2-geodesics, $g_{i}$ and $g_{i+l}$ travel a $\Gamma$-distance bounded by $c(2)$ in each coset they penetrate. By Lemma 5.7 case (11), $l_{\Gamma}\left(h_{2}\right) \leq l_{\Gamma}\left(h_{1}\right)+2 c(2)$ and $l_{\Gamma}\left(h_{3}\right) \leq l_{\Gamma}\left(h_{1}\right)+4 c(2)$, so that in what follows, we assume that $i \geq 4$. To show that the $\Gamma$-length of $h_{i}$ is bounded, we glue $i-2$ consecutive $G$-floors of the cascade along $g_{j}$ where $j=2,3, \ldots, i-2$, and $i-1$ consecutive $H$-floors of the cascade along $h_{j}$, for $j=2,3, \ldots, i-1$. We have the following equalities:

$$
\begin{aligned}
& k_{1} k_{2} \ldots k_{i-2}=g_{1} c_{2} c_{3} \ldots c_{i-1} g_{i-1}^{-1} \\
& k_{1} k_{2} \ldots k_{i-2}=h_{1}^{-1} c_{1} c_{2} \ldots c_{i-2} c_{i-1} h_{i} k_{i-1}^{-1}
\end{aligned}
$$

Denote $\bar{c}=c_{2} c_{3} \ldots c_{i-1}$ and $\bar{h}=h_{1}^{-1} c_{1} \bar{c} h_{i} k_{i-1}^{-1}$; it follows from the equalities above that $g_{1} \bar{c} g_{i-1}^{-1}=\bar{h}$. Therefore, $g_{1} \bar{c}$ and $\bar{h} g_{i-1}$ form a pair of 2-quasigeodesics with common endpoints. Hence, the BCP property implies that the $\Gamma$-length of both $\bar{c}$ and $\bar{h}$ is bounded by $c(2)$. Since $h_{i}=\left(c_{1} \bar{c}\right)^{-1} h_{1} \bar{h} k_{i-1}$, according to Lemma 5.7 we have that $l_{\Gamma}\left(h_{i}\right) \leq l_{\Gamma}\left(h_{1}\right)+C_{0}+3 c(2)$.

\subsection{Relatively short conjugating elements are short}
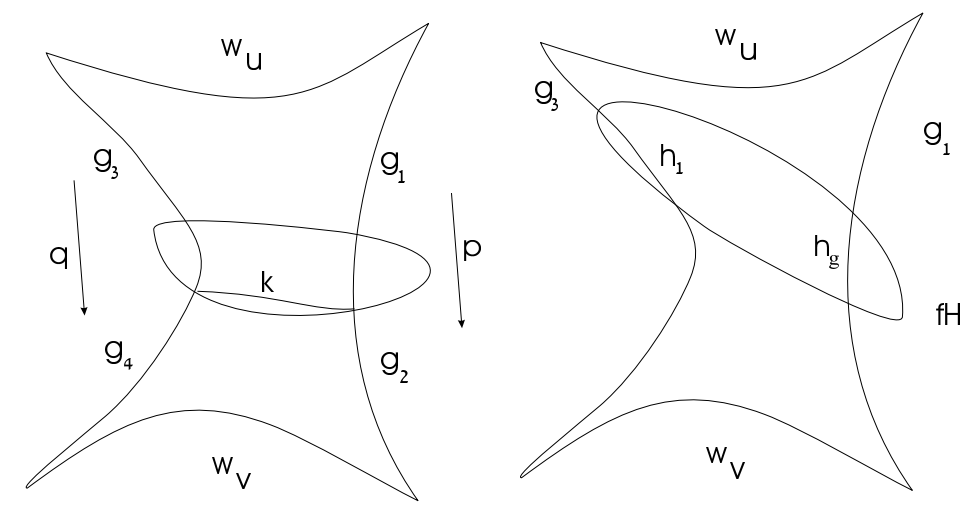

Figure 4: Illustrated above is Lemma [5.9] case (5), when $\hat{p}$ and $\hat{q}$ penetrate a coset $f H$. 
The following lemma enables one to bound the $\Gamma$-length of $g$ in terms of the relative length of $\hat{g}, \hat{u}$ and $\hat{v}$.

Lemma 5.9 Let $\hat{p}$ penetrate a left coset $f H$ when moving along an $H$ geodesic $h_{g}$. Assume that $\hat{w}$ backtracks to $f H$.

(1) If $\hat{p}, \hat{w}_{u}, \hat{q}$ and $\hat{w}_{v}$ all penetrate the coset $f H$, then either

(a) $u$ and $v$ are conjugate in $G$ to $k \in H$ with the length bounded as follows: $l_{\Gamma}(k) \leq Q+2 C$, or

(b) $l_{\Gamma}\left(h_{g}\right) \leq Q+2 C$.

(2) If $\hat{p}, \hat{w}_{u}$ and $\hat{q}$ penetrate $f H$, and $\hat{w}_{v}$ does not penetrate it, then either

(a) $u$ and $v$ are conjugate in $G$ to $k \in H$ with $l_{\Gamma}(k) \leq C$, or

(b) $l_{\Gamma}\left(h_{g}\right) \leq Q+3 C+3 c(2)$.

(3) If $\hat{p}, \hat{w}_{u}$ and $\hat{w}_{v}$ penetrate $f H$, and $\hat{q}$ does not penetrate it, then $l_{\Gamma}\left(h_{g}\right) \leq$ $2 Q+3 C$.

(4) If $\hat{p}$ and $\hat{w}_{u}$ penetrate a coset $f H$, and neither $\hat{w}_{v}$ nor $\hat{q}$ penetrates $f H$, then $l_{\Gamma}\left(h_{g}\right) \leq Q+2 C$.

(5) If $\hat{p}$ and $\hat{q}$ penetrate $f H$, and neither $\hat{w}_{u}$ nor $\hat{w}_{v}$ penetrates it, then either

(a) $u$ and $v$ are conjugate in $G$ to $k \in H$ with $l_{\Gamma}(k) \leq C$, or

(b) $l_{\Gamma}\left(h_{g}\right) \leq Q+3 C+3 c(2)$

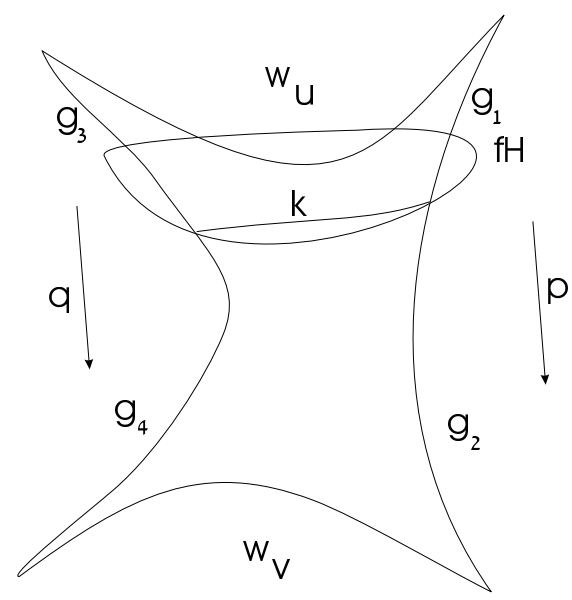

Figure 5: Illustrated above is Lemma [5.9] case (2), when $\hat{p}, \hat{w}_{u}$ and $\hat{q}$ penetrate a coset $f H$, and $u$ and $v$ are conjugate to $k$. 
Proof Case (3) is a particular case of Corollary 5.4. The statement in the case (4) follows from Lemma 5.3. In the proof below, we use the following notation. Let $g=g_{1} h_{g} g_{2}$ (we denote by $g_{i}$ the sub-paths of $p$ and also their labels), and let $f H$ be the coset in which $\hat{p}$ travels along $h_{g}$. In the case (5), $\hat{q}$ penetrates the coset $f H$ also; denote by $h_{f}$ the $H$-geodesic in $f H$, along which $\hat{q}$ moves there. Therefore, $g=g_{3} h_{f} g_{4}$, where $g_{3}, g_{4}$ denote both sub-paths of $q$ and their labels. If $l_{\hat{\Gamma}}\left(\hat{g_{1}}\right)=l_{\hat{\Gamma}}\left(\hat{g_{3}}\right)$ (Figure 4, left), then necessarily $g_{1}=g_{3}, h_{g}=h_{f}$, and $g_{2}=g_{4}$. Let $k$ be a $H$-geodesic joining the points where $p$ and $q$ last exit $f H$. We have that $k=h_{g}^{-1} g_{1}^{-1} u g_{1} h_{g}$, and $k=g_{2} v g_{2}^{-1}$. Moreover, the closed path $g_{2} w_{v} g_{2}^{-1} k$ has length less than $L$ and satisfies the conditions of Lemma 5.2 Hence, we obtain (5a). Now, assume that $l_{\hat{\Gamma}}\left(\hat{g_{1}}\right)>l_{\hat{\Gamma}}\left(\hat{g_{3}}\right)$ (Figure 4 right). Let $k_{1}$ (or $k_{2}$ ) be a geodesic joining the points where $p$ and $q$ first enter (or last exit) $f H$; both closed paths (one goes through $k_{1}$ and $w_{u}$ and the other one goes through $k_{2}$ and $w_{v}$ ) that we obtain, are shorter than $w$ and satisfy the conditions of Lemma 5.2. Hence, $\left|l_{\Gamma}\left(h_{f}\right)-l_{\Gamma}\left(h_{g}\right)\right| \leq 2 C$. Furthermore, $h_{f}$ is a subword of $g_{1}$. Let $f_{1} H$ be the coset that $\hat{p}$ penetrates along $h_{f}$. If $\hat{w}$ does not backtrack to $f_{1} H$, then one can apply Lemma 5.2 and obtain that $l_{\Gamma}\left(h_{f}\right) \leq C$. If $\hat{w}$ backtracks to $f_{1} H$ but in $\hat{w}$ cascade effect does not occur, then we apply either Lemma 5.5 or Lemma 5.3 and conclude that $l_{\Gamma}\left(h_{f}\right) \leq l_{\Gamma}(v)+C+c(2)$, so that $l_{\Gamma}\left(h_{g}\right) \leq l_{\Gamma}(v)+3 C+c(2)$. If in $\hat{w}$ cascade effect occurs, then we apply Lemma 5.8, and Lemma 5.3 to show that $l_{\Gamma}\left(h_{g}\right) \leq\left(l_{\Gamma}(v)+C+c(2)\right)+2 C+2 c(2)$. In any case, we get the statement of the case (5b).

Case (2) If $l_{\Gamma}\left(g_{1}\right)=l_{\Gamma}\left(g_{3}\right)$ (Figure 5), then both $u$ and $v$ are conjugate to $k \in H$ with $l_{\Gamma}(k) \leq C$, and we obtain (2a). Let $l_{\hat{\Gamma}}\left(\hat{g}_{1}\right)>l_{\hat{\Gamma}}\left(\hat{g}_{3}\right)$ (Figure 6] left). Assume that $\hat{p}, \hat{w}_{u}$ and $\hat{q}$ penetrate another coset $f_{1} H$ so that $\hat{q}$ travels in $f_{1} H$ along $h_{g}$. As both $\hat{w}_{u}$ and $\hat{p}^{-1}$ are geodesics, the distances from their common terminal point to a coset they both penetrate, are equal; therefore, the case shown on Figure [6] left, is the only possible one. In this case, the argument used to prove Lemma [5.5] implies that $l_{\Gamma}\left(h_{g}\right) \leq C$. The other cases (Figure [6] right, shows one of those) are analogous to the case (5b), so that $l_{\Gamma}\left(h_{g}\right) \leq l_{\Gamma}(v)+3 C+3 c(2)$ which proves (2b).

Case (11) follows easily from the above arguments. If $l_{\hat{\Gamma}}\left(\hat{g}_{1}\right)=l_{\hat{\Gamma}}\left(\hat{g}_{3}\right)$ (Figure 7 left) then we have the case (1a). Indeed, it follows that $g_{1}=g_{3}$ and $g_{2}=g_{4}$. Let $k$ be a $\Gamma$-geodesic joining the points where $p$ and $q$ leave $f H$; hence, both $u$ and $v$ are conjugate to $k$ and $l_{\Gamma}(k)<l_{\Gamma}(v)+2 C$, as claimed. In the other cases (see, for instance, Figure 6] right, where $\left.l_{\hat{\Gamma}}\left(\hat{g}_{1}\right)<l_{\hat{\Gamma}}\left(\hat{g}_{3}\right)\right)$, Lemma 5.3 implies the statement of (1b).

Corollary 5.10 If $u g v g^{-1}=1$ in $G$, then either $u$ and $v$ are conjugate in $G$ 

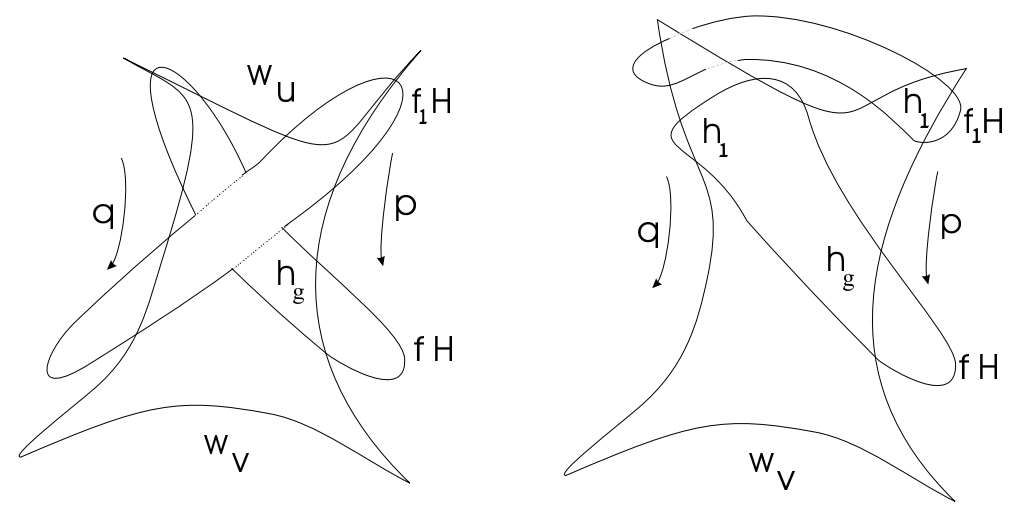

Figure 6: Illustrated above is Lemma [5.9 case (2), when $\hat{p}, \hat{w}_{u}$ and $\hat{q}$ penetrate two cosets of $H$.
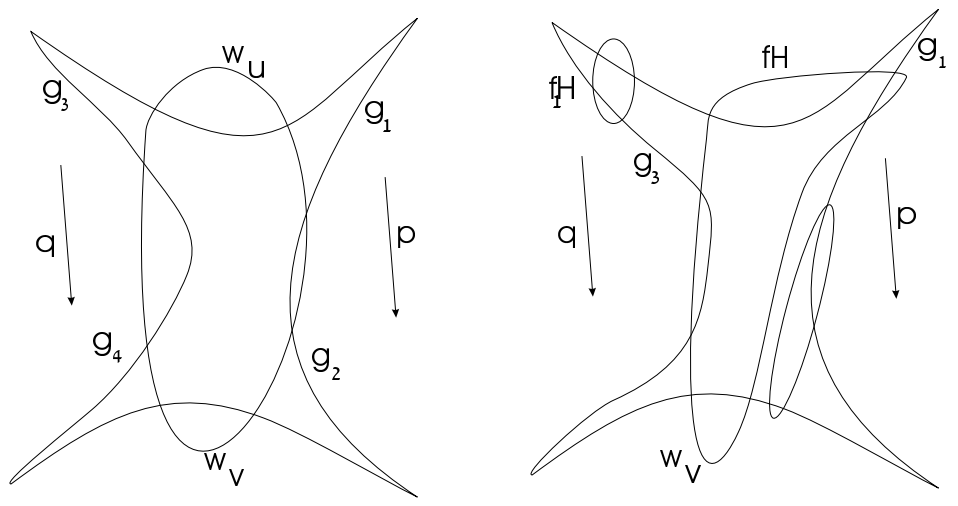

Figure 7: Illustrated above is Lemma [5.9, case (1), when $\hat{p}, \hat{w}_{u}, \hat{q}$ and $\hat{w}_{v}$ penetrate a coset of $H$.

to $k \in H$ with $l_{\Gamma}(k) \leq Q+2 C$, or $g$ travels a $\Gamma$-distance bounded by $2 Q+6 C$, in each coset it penetrates.

\subsection{A global bound on the length of $g$}

We have proved that if the relative length of $g$ is bounded, then the $\Gamma$-length of $g$ can be bounded as well. A priori, we do not have any bound on the relative length of $g$. It turns out that in order to bound globally the relative length of $g$ (see the proof of Theorem 5.12 below), we need to bound distances which $g$ travels in $H$-cosets. Let $D=c(8 Q)$.

Lemma 5.11 If $u_{g v g}{ }^{-1}=1$ in $G$, then either 
(1) $u$ and $v$ are conjugate in $G$ to an element $k \in H$, or

(2) The $\Gamma$-distance which $g$ travels in a coset it penetrates, is bounded by $l_{H}^{g}=2 Q+10 D$.

Proof Assume that the conditions of the case (11) do not hold. Let $h_{g}$ be an $H$-subword of $g$ of the maximal possible length.

First, assume that the path $h_{g}$ is not a subpath of an $H$-floor of a cascade. We claim that in this case the $\Gamma$-length of $h_{g}$ satisfies the following inequality:

$$
l_{\Gamma}\left(h_{g}\right) \leq 2 Q+6 D .
$$

Our proof of this latter claim splits according to the following possibilities.

(1) If $l_{\hat{\Gamma}}(\hat{g})<3 Q$, then $l_{\hat{\Gamma}}(\hat{w})<8 Q$, and by Corollary 5.10, we conclude that the inequality (5) holds.

(2) If $l_{\hat{\Gamma}}(\hat{g}) \geq 3 Q$, then by Lemma 4.2, $\lambda_{1}$ and $\lambda_{2}$ form a pair of $(2 \hat{Q}+1)$ quasi-geodesics with common endpoints. We distinguish the following two cases:

(a) If $\hat{p}$ and $\hat{q}$ do not penetrate the same coset, then by Corollary 5.4 $l_{\Gamma}\left(h_{g}\right) \leq 2 Q+c(2 \hat{Q}+1)+2 c(2)$. As $c(2 \hat{Q}+1)<D$ and $c(2)<D$, it follows that $l_{\Gamma}\left(h_{g}\right)$ satisfies the inequality (15).

(b) If $\hat{p}$ and $\hat{q}$ penetrate the same coset but $h_{g}$ is not a subpath of an $H$-floor of the cascade, then one can find a closed path $\hat{w}^{\prime}$ which goes through $h_{g}$ and so that the subsegments of $\hat{p}$ and of $\hat{q}$ which belong to this closed path, do not penetrate the same coset. Therefore, the arguments used in the cases (11) and (2a) apply to $\hat{w}^{\prime}$, so that the inequality (15) holds in this case also. Observe that if in $\hat{w}$ the cascade effect of length $n$ occurs, then $h_{1}$ and $h_{n+1}$ occur also outside the cascade. Hence, the argument that we use in this case, applies to $h_{1}$ and $h_{n+1}$ as well. Thus, both $l_{\Gamma}\left(h_{1}\right)$ and $l_{\Gamma}\left(h_{n+1}\right)$ satisfy the inequality (5).

Now, assume that $h_{g}$ is a subpath of an $H$-floor of a cascade. By Lemma 5.8 $l_{\Gamma}\left(h_{g}\right) \leq l_{\Gamma}\left(h_{1}\right)+2 C_{0}+2 c(2)$. Since $l_{\Gamma}\left(h_{1}\right) \leq 2 Q+6 D$ (see the the proof in the case (2b) above), and $C_{0}<D$, we have that $l_{\Gamma}\left(h_{g}\right) \leq 2 Q+10 D$.

The following theorem establishes explicitly the dichotomy mentioned above: either $u$ and $v$ are conjugate to an element of $H$, or the $\Gamma$-length of $g$ can be globally bounded. 
Theorem 5.12 Let $G$ be a group hyperbolic relative to a subgroup $H$, in the strong sense. If $u g v g^{-1}=1$ in $G$ and the relative length of $g$ is positive and minimal possible, then either

(1) $u$ and $v$ are conjugate in $G$ to an element $k \in H$ so that $g=g_{1} g_{2}$ where $u=g_{1} k g_{1}^{-1}$ and $k=g_{2} v g_{2}^{-1}$, or

(2) The $\Gamma$-length of $g$ is bounded in terms of the $\Gamma$-lengths of $u$ and $v$.

Proof We assume that the case (1) does not occur. Let $\hat{\gamma}$ be a geodesic joining $\hat{p}(t)$ and $\hat{q}(t)$, and let $m=l_{0}+2 K(\mathrm{cf}$. (4) $)$ be the upper bound for the $\hat{\Gamma}$-length of $\hat{\gamma}$ obtained in the proof of Lemma 4.5. Assume that $l_{\hat{\Gamma}}(\hat{g})>2 \hat{Q}+2 \delta+6 m$ so that we can consider values of $t$ satisfying the inequality $t_{1}+3 m<t<t_{2}-3 m$ $\left(t_{1}, t_{2}\right.$ are as in Lemma 4.5). Denote by $\hat{\gamma}_{1}$ a geodesic joining $\hat{p}(t+3 m)$ and $\hat{q}(t+3 m)$, and denote by $\hat{\gamma}_{p}$ (or $\hat{\gamma}_{q}$ ) the segment of $\hat{p}$ (or $\hat{q}$ ) between $\hat{p}(t)$ (or $\hat{q}(t))$ and $\hat{p}(t+3 m)$ (or $\hat{q}(t+3 m))$. The closed path $\hat{w}=\hat{\gamma}_{p} \hat{\gamma}_{1} \hat{\gamma}_{q}^{-1} \hat{\gamma}^{-1}$ has a relative length bounded by $8 m$. Moreover, since $l_{\hat{\Gamma}}(\hat{\gamma}), l_{\hat{\Gamma}}\left(\hat{\gamma}_{1}\right) \leq m$ and the distance between their initial (or terminal) points equals $3 m$, these two geodesics never penetrate the same coset. Therefore, by Corollary [5.4 the maximal distance that $\hat{\gamma}$ (or $\hat{\gamma}_{1}$ ) can travel in a coset it penetrates, is bounded above by $2 l_{H}^{g}+3 c(8 m)$. Therefore, the $\Gamma$-length of $\gamma$ is bounded in terms of the $\Gamma$-lengths of $u$ and $v$, so that we can apply the argument used to prove Theorem [4.6] This argument tells that since $l_{\hat{\Gamma}}(\hat{g})$ is minimal possible, it is bounded in terms of the $\Gamma$-lengths of $u$ and $v$. By Lemma 5.11 one obtains the claim.

\subsection{The case when $u$ and $v$ are conjugate to an element of $H$}

In general, there is a finite sequence of elements $k_{1}, k_{2}, \ldots, k_{n}$ of $H$ conjugate in $G$ to each other as follows: $k_{i}=g_{i} k_{i+1} g_{i}^{-1}$, so that $u=g_{u} k_{1} g_{u}^{-1}$ and $v=g_{v} k_{n} g_{v}^{-1}$ for some $g_{i}, g_{u}$ and $g_{v}$ in $G$. We are able to find bounds for the $\Gamma$-length of $g_{u}$, of $g_{v}$ and of those $g_{i}$ which are in $G \backslash H$, but if $g_{i} \in H$, then its $\Gamma$-length cannot be bounded. Therefore, there is no bound on the $\Gamma$-length of the element $g=g_{u} g_{1} \ldots g_{n-1} g_{v}$ conjugating $u$ and $v$. Note that in the geometric picture, $\hat{p}$ and $\hat{q}$ penetrate the same cosets, at the same moments of time, so that $\hat{w}$ is a finite sequence of digons with two isosceles triangles at both ends of it. In the case of a hyperbolic groups, this picture would mean that both $u$ and $v$ were conjugate to the trivial element, therefore, were trivial elements themselves.

Our approach is as follows. Lemma 5.13 implies that the $\Gamma$-length of $k_{1}$ and $k_{n}$ can be bounded in terms of the length of $u$ and of $v$. By Corollary 5.15. 
$l_{\Gamma}\left(k_{i}\right) \leq c(2)$ for $1<i<n$. Moreover, the length of $g_{u}$, of $g_{v}$ and of those conjugating elements $g_{i}$ which are not in $H$, can be bounded in terms of the length of $u$ and of $v$ as well. Therefore, it is enough to consider the finite set $H_{d}$ of elements of $H$ whose length does not exceed $d=c(2)$. Lemma [5.16] below allows one to obtain the partition of $H_{d}$ to conjugacy classes of $G$. Having obtained this partition, we are able to establish whether or not $u$ and $v$ are conjugate to each other, if each one of them is conjugate to an element of $H$.

Lemma 5.13 Let $u \in G$ be conjugate to $h \in H$. Then either $u \in H$, and $u$ and $h$ are conjugate in $H$, or there exist $k \in H$ and $g \in G$ so that $u=g k g^{-1}$ and the following conditions hold:

(1) If $f \in G$ and $k_{f} \in H$ satisfy the equality $u=f k_{f} f^{-1}$, then $l_{\hat{\Gamma}}(\hat{g}) \leq l_{\hat{\Gamma}}(\hat{f})$.

(2) $l_{\Gamma}(k) \leq C_{0}$.

(3) $l_{\Gamma}(g)$ is bounded in terms of the $\Gamma$-length of $u$.

Proof Assume that $u \notin H$. Corollary 3.2 implies that $g \in G \backslash H$, in particular the relative length of $g$ is strictly positive. Therefore, the minimal possible relative length is attained, and we get (11). Fix an element $g$ that satisfies the condition (11), and consider the closed path $w=u g k g^{-1}$ and its projection $\hat{w}$ into $\hat{\Gamma}$. By Lemma 4.2, either $l_{\hat{\Gamma}}(\hat{w}) \leq 7 \hat{Q}$, or $\hat{w}$ is the concatenation of two $(2 \hat{Q}+1)$-quasi-geodesics. The assertion (2) follows then either from Lemma 5.2 , or from the definition of the BCP property. To obtain the assertion (3), apply Theorem 5.12 and note that the assertion (1) we have just proven, implies that the case (11) mentioned in the statement of Theorem 5.12 does not occur.

Corollary 5.14 Given $u \in G \backslash H$, one can determine effectively, whether or not there is $h \in H$ so that $u$ is conjugate to $h$.

Proof According to Lemma 5.13, it is enough to determine whether or not the word $u g h g^{-1}$ is trivial for some $h$ and $g$ whose $\Gamma$-length is bounded. There are only finitely many possibilities to choose $h$ and $g$, and by [14, Theorem 3.7] (see Theorem 1.2), for each particular choice of these elements, an answer can be found effectively.

Corollary 5.15 If $u \in H$ and $h \in H$ are conjugate in $G$ but are not conjugate in $H$, then the assertion (2) of Lemma 5.13] becomes $l_{\Gamma}(k) \leq c(2)$.

Proof Note that in this case the projection $\hat{w}$ of the closed path $w=u g k g^{-1}$ is the concatenation of two 2-quasi-geodesics. 
Lemma 5.16 Given $h_{u}, h_{v} \in H$, one can determine effectively whether or not $h_{u}$ and $h_{v}$ are conjugate in $G$.

Proof First, we check whether or not $h_{u}$ and $h_{v}$ are conjugate in $H$. Assume that this is not the case. Let $d=c(2)$ be the constant given by Definition 2.3. Consider the finite subset $H_{d}=\left\{h \in H \mid l_{\Gamma}(h) \leq d\right\}$ of "short" elements of $H$, and the partition of $H_{d}$ into conjugacy classes $\mathcal{C}_{G}$ of $G$ : elements $h_{1}$ and $h_{2}$ of $H_{d}$ belong to a $\mathcal{C}_{G}$-class if and only if there is $g \in G$ such that $h_{v}=g h_{u} g^{-1}$. We claim that this partition of $H_{d}$ can be obtained in a finite time. Indeed, as the conjugacy problem in $H$ is solvable, we can find a partition $\mathcal{C}_{H}$ of $H_{d}$ into conjugacy classes of $H$ in a finite time. Furthermore, we define bounded $\mathcal{C}_{G}$-classes as follows: elements $\tilde{k}$ and $\tilde{h}$ of $H_{d}$ belong to a bounded $\mathcal{C}_{G}$-class if and only if either $\tilde{k}$ and $\tilde{h}$ belong to a $\mathcal{C}_{H}$-class, or there is a finite sequence $\tilde{k}=k_{1}, k_{2}, \ldots, k_{n}=\tilde{h}$ of elements of $H$ with $l_{\Gamma}\left(k_{j}\right) \leq c(2)$, so that for every $i=1,2, \ldots, n-1$ there is $g_{i} \in G$ with bounded length $l_{\Gamma}\left(g_{i}\right)$ such that $h_{i}=g_{i} h_{i+1} g_{i}^{-1}$. Corollary 5.15 implies that two elements of $H_{d}$ belong to a $\mathcal{C}_{G}$-class if and only if they belong to a bounded $\mathcal{C}_{G}$-class. This observation gives rise to the following algorithm. Pick a $\mathcal{C}_{H}$-class $H_{d}^{(1)}=\left\{h_{1}^{(1)}, \ldots, h_{m_{1}}^{(1)}\right\}$. For each $h_{i}^{(1)} \in H_{d}^{(1)}$, find all those pairs of elements $k \in H$ and $g \in G \backslash H$ which satisfy the conditions of Lemma 5.13 and Corollary [5.15. Since each $k$ is a "short" element of $H$, it belongs to a $\mathcal{C}_{H}$-class $H_{d}^{(j)}$. We add all these classes $H_{d}^{(j)}$ to $H_{d}^{(1)}$ so as to obtain a bounded $\mathcal{C}_{G}$-class, and declare all these added elements as new members in the $\mathcal{C}_{G}$-class. Having collected all $k$ and their $\mathcal{C}_{H^{-}}$ classes, we repeat the above procedure for each new member in the $\mathcal{C}_{G}$-class of $h_{u}$. Again, added elements are declared to be new members, and we proceed with them in the same manner, until there are no new members anymore. Then we pick a $\mathcal{C}_{H}$-class, which is not a subset of the bounded $\mathcal{C}_{G}$-class of $h_{u}$ we have just obtained, and repeat the same procedure. The algorithm stops when the (finite) set of $\mathcal{C}_{H}$-classes is exhausted.

Having obtained the partition of $H_{d}$ into $\mathcal{C}_{G}$-classes, we check whether or not there are $k_{u}, k_{v} \in H_{d}$ that belong to same $\mathcal{C}_{G}$-class and such that $k_{u}$ and $h_{u}$ as well as $k_{v}$ and $h_{v}$, are conjugate in $H$ by elements of bounded length (see the assertion (3) of Lemma 5.13). The elements $h_{u}$ and $h_{v}$ are conjugate in $G$ if and only if there are $k_{u}$ and $k_{v}$ as above.

From Corollary 5.14 and Lemma 5.16 we obtain the following corollary.

Corollary 5.17 Given $u \in G \backslash H$ and $h \in H$, one can determine effectively, whether or not $u$ and $h$ are conjugate in $G$. 


\subsection{Proof of Theorem 1.1}

Proof By Corollary 3.2 we can determine whether or not $u$ and $v$ belong to $H$. If both $u$ and $v$ are in $H$, then the assertion follows from Lemma 5.16. If for instance $u \in G \backslash H$ while $v \in H$, then the assertion follows from Corollary 5.17 . Now, assume that neither $u$ nor $v$ is in $H$. According to Corollary 5.14 we can answer effectively the following two questions:

(1) Is there $k_{u} \in H$ so that $k_{u}$ and $u$ are conjugate in $G$ ?

(2) Is there $k_{v} \in H$ so that $k_{v}$ and $v$ are conjugate in $G$ ?

If the answers are different, then $u$ and $v$ are not conjugate in $G$. If both answers are positive, then we apply Lemma [5.16 to $k_{u}$ and $k_{v} ; u$ and $v$ are conjugate in $G$ if and only if $k_{u}$ and $k_{v}$ are conjugate in $G$. If both answers are negative, then by Theorem [5.12, $u$ and $v$ are conjugate if and only if there is a conjugating element of a bounded $\Gamma$-length. Since balls of bounded radii in the Cayley graph $\Gamma$ of $G$ are compact, this latter condition can be checked effectively.

\subsection{Group with several parabolic subgroups}

The definition of a relatively hyperbolic group can be extended to the case of several subgroups [14, Section 5]. Let $G$ be a group, and let $\left\{H_{1}, \ldots, H_{r}\right\}$ be a finite set of finitely generated subgroups of $G$. In the Cayley graph of $G$, for every $i=1,2, \ldots, r$, add a vertex $v\left(g H_{i}\right)$ for each left coset of $H_{i}$ in $G$, and connect this new vertex (by an edge with length $\frac{1}{2}$, as before) with each element of this left coset. This new graph $\hat{\Gamma}$ is called the coned-off graph of $G$ with respect to $\left\{H_{1}, \ldots, H_{r}\right\}$. The group $G$ is weakly hyperbolic relative to $\left\{H_{1}, \ldots, H_{r}\right\}$, if $\hat{\Gamma}$ is a hyperbolic metric space. The definition of the BCP property can be extended in an obvious way to this case. If the subgroups $H_{1}, \ldots, H_{r}$ are torsion-free, then the BCP property implies that these subgroups are pairwise conjugacy separated. This means that if $g H_{i} g^{-1} \cap H_{j} \neq \emptyset$ for some $g \in G, 1 \leq i, j \leq r$, then necessarily $i=j$ and $g \in H_{i}$. The group $G$ is strongly hyperbolic relative to the family of subgroups $\left\{H_{1}, \ldots, H_{r}\right\}$, if $G$ is weakly hyperbolic relative to $\left\{H_{1}, \ldots, H_{r}\right\}$, and the pair $\left(G,\left\{H_{1}, \ldots, H_{r}\right\}\right)$ has the BCP property.

Our arguments can easily be extended to prove the following generalization of Theorem 1.1 . 
Theorem 5.18 Let $G$ be a group strongly hyperbolic relative to a finite set of subgroups $\left\{H_{1}, \ldots, H_{r}\right\}$. If the conjugacy problem is solvable in $H_{i}$ for all $i=1,2, \ldots, r$, then it is solvable in $G$.

\section{Fundamental groups of negatively curved mani- folds}

In this section, we prove Theorem 1.3. Let $G$ be the fundamental group of a negatively curved non-compact manifold of finite volume with a single cusp, and let $H$ denote the cusp subgroup of $G$. Then $G$ is hyperbolic relative to $H$ in the strong sense (Farb 14] gave a direct proof of this assertion). Since $H$ is a nilpotent group, the conjugacy problem for $H$ is solvable [25]. Therefore, in order to prove Theorem [1.3 it remains to show that the constants $c(P)$ in Definition 2.3 can be bounded effectively.

We follow [14 and 29]. $\tilde{M}$ denotes a Hadamard manifold; we are most interested in the case when $\tilde{M}$ is the universal cover of a complete, finitevolume negatively curved Riemannian manifold $M$ with pinched negative curvature $-b^{2} \leq K(M) \leq-a^{2}<0$. Our calculation is based on the geometry of horospheres in $\tilde{M}$. Let $x \in \tilde{M}, z$ be a point at infinity, and let $\gamma$ be the geodesic ray from $x$ to $z$. Horospheres are the level surfaces of the Busemann function $F=\lim _{t \rightarrow \infty} F_{t}$, where $F_{t}$ is defined by $F_{t}(p)=d_{\tilde{M}}(p, \gamma(t))-t$. Let $S$ be a horosphere, we denote by $d_{S}$ the induced path metric on $S$; that is, $d_{S}(x, y)$ is the infimum of the length of all paths in $S$ from $x$ to $y$.

Proposition 6.1 14, Proposition 4.2] If $\gamma$ is a geodesic tangent to $S$, and $p$ and $q$ are projections of $\gamma( \pm \infty)$ onto $S$, then

$$
\frac{2}{b} \leq d_{S}(p, q) \leq \frac{2}{a}
$$

Proposition 6.2 (29, Corollary 5.3], cf. [14, Proposition 4.3]) Let $S$ and $S^{\prime}$ be nonintersecting horospheres based at distinct points of $\partial H$. Then the $S$-diameter of the projection $\pi_{S}\left(S^{\prime}\right)$ is at most $\frac{4}{a}+2 \delta$, where $\delta$ is the Gromov hyperbolicity constant for $\tilde{M}$.

Definition 6.3 14] Let $\gamma$ be a geodesic in $\tilde{M}$ not intersecting a horosphere $S$. Given $s \in S$, we say that $\gamma$ can be seen from $s$ if $\overline{s \gamma(t)} \cap S=\{s\}$ for some $t$. Let $T_{\gamma}$ be the set of points $s \in S$ that $\gamma$ can be seen from. The visual size $V_{S}$ of the horosphere $S$ is defined to be the supremum of the diameter of $T_{\gamma}$ in the 
metric $d_{S}$, where the supremum is taken over all geodesics $\gamma$ not intersecting $S$.

Proposition 6.4 (29, Lemma 5.4], cf. [14, Lemma 4.4]) Horospheres in a pinched Hadamard manifold have (uniformly) bounded visual size.

The proof shows that the visual size of $S$ is bounded by $\frac{2}{a}+C$ where according to [29. Lemma 4.10], $C=2 \delta+\log 16$. Therefore, the visual size $V_{S}$ of $S$ satisfies the following inequality:

$$
V_{S} \leq \frac{2}{a}+2 \delta+\log 16
$$

Let $G$ be the fundamental group of $M$ so that $M=\tilde{M} / G$. We can choose a $G$-invariant set of horoballs so that there is a uniform lower bound on the distance between horoballs and the action of $G$ on the horoballs has finitely many orbits. Having deleted the interiors of all of these horoballs, we obtain a space $X$ on which $G$ acts cocompactly by isometries ( $X$ is equipped with the path metric). Choose a base point $x \in X$, the map $g \mapsto g \cdot x$ gives a quasi-isometry $\psi: \Gamma \longrightarrow X$ of the Cayley graph of $G$ with $X$; for each coset $g H$, all of the elements of $g H$ are mapped to the same horosphere. The electric space $\hat{X}$ is the quotient of $X$ obtained by identifying points which lie in the same horospherical boundary component of $X$. The quotient $\hat{X}$ has a path pseudo-metric $d_{\hat{X}}$ induced from the path metric $d_{X}$; the pseudo-metric $d_{\hat{X}}$ can be thought of as a pseudo-metric on $X$, where the distance between two points is the length of the shortest path between them, but path-length along a horosphere $S \subset \partial X$ is measured as zero length. Locally $d_{\hat{X}}$ agrees with $d_{\tilde{M}}$ on the interior of $X$.

Proposition 6.5 [14, Proposition 4.6] The electric space $\hat{X}$ is a $\delta^{\prime}$-hyperbolic pseudometric space for some $\delta^{\prime}>0$.

Given a path $\gamma$ in $\hat{X}$, the electric length $l_{\hat{X}}(\gamma)$ is the sum of the $X$-length of subpaths of $\gamma$ lying outside every horosphere. An electric geodesic between $x, y \in \hat{X}$ is a path $\gamma$ in $\hat{X}$ from $x$ to $y$ such that $l_{\hat{X}}(\gamma)$ is minimal. An electric $P$-quasi-geodesic is a $P$-quasi-geodesic in $\hat{X}$.

Lemma 6.6 (29, Lemma 5.6], cf. [14, Lemma 4.5]) Given $P>0$, there exist constants $K=K(P), L=L(P)>0$ such that for any electric $P$-quasigeodesic $\beta$ from $x$ to $y$, if $\gamma$ is the $\tilde{M}$ geodesic from $x$ to $y$, then $\beta$ stays completely inside $N b h d_{\hat{X}}(\gamma, K+L / 2)$. 
According to the proof, $K$ can be chosen so that

$$
K \geq \frac{1}{a} \log \left(2 P\left(V_{S}+1\right)\right) .
$$

Then one can set

$$
L=4 P K\left(2+V_{S}\right)+8 P \delta
$$

where $V_{S}$ is as in (6).

Lemma 6.7 (29, Lemma 5.7], cf. [14, Lemma 4.7]) Let $\beta$ be an electric $P$ quasi-geodesic so that $\beta \cap S=\emptyset$. Then there exists a constant $D=D(P)$ so that $\pi_{S}(\beta)$ has $S$-length at most $D l_{\hat{X}}(\beta)$.

The proof shows that

$$
D=1+V_{S} \leq 1+\frac{2}{a}+2 \delta+\log 16 .
$$

Lemma 6.8 (29, Lemma 5.9], cf. [14, Lemma 4.8 and Lemma 4.9]) Let $\alpha$ and $\beta$ be electric $P$-quasi-geodesics from $x$ to $y$ in $\hat{X}$. Then there exists a constant $E$ such that the following conditions hold:

(1) Suppose $\alpha$ first greets $S$ at $\alpha\left(s_{0}\right)$ and $\beta$ first greets $S$ at $\beta\left(t_{0}\right)$. Suppose that $\alpha$ and $\beta$ leave $S$ at $\alpha\left(s_{1}\right)$ and $\beta\left(t_{1}\right)$. Then

$$
d_{S}\left(\alpha\left(s_{0}\right), \beta\left(t_{0}\right)\right)<E \quad \text { and } \quad d_{S}\left(\alpha\left(s_{1}\right), \beta\left(t_{1}\right)\right)<E .
$$

(2) Suppose $\alpha$ greets $S$ at $\alpha\left(s_{0}\right)$ and leaves $S$ at $\alpha\left(s_{1}\right)$. Suppose that $\beta$ doesn't greet $S$. Then $d_{S}\left(\alpha\left(s_{0}\right), \alpha\left(s_{1}\right)\right)<E$.

By the proof, $E=3 \max \{\delta, D\} P(K+L)$, where $D, K$ and $L$ are given by (9), (77) and (8), respectively. Altogether, we have

$$
E \leq 3 P\left(1+V_{S}\right)(K+L),
$$

where $V_{S}$ is as in (6). Therefore, the upper bound for the constant $E$ can be computed effectively. Let $\lambda$ denote the quasi-isometry constant of the map $\psi: \Gamma \longrightarrow X$. Then the constants $c(P)$ of Definition 2.3 can be bounded as follows: $c(P) \leq \lambda E$.

Acknowledgements The first version of this paper was written when I was a postdoctoral fellow at the Technion, Haifa. I am thankful to Arye Juhász for stimulating discussions, and to Benson Farb for many helpful electronic communications. Also, I want to thank Michah Sageev for pointing me out the example of a weakly relatively hyperbolic group with unsolvable conjugacy problem. Finally, I am deeply grateful to the referee for extremely useful remarks and helpful suggestions. 


\section{References}

[1] E Alibegovic, A combination theorem for relatively hyperbolic groups, Bull. London Math. Soc. (to appear)

[2] J M Alonso, T Brady, D Cooper et al, Notes on word hyperbolic groups, from: "Group theory from a geometrical viewpoint", (E Ghys, A Haefliger, A Verjovsky, editors) ICTP, Trieste (1990) 3-63 MathReview

[3] B Baumslag, Residually free groups, Proc. London Math. Soc. 17 (1967) 402418 MathReview

[4] B H Bowditch, Relatively hyperbolic groups, preprint, University of Southampton (1998)

[5] B H Bowditch, Connectedness properties of limit sets, Trans. Amer. Math. Soc. 351 (1999) 3673-3686 MathReview

[6] B H Bowditch, Boundaries of geometrically finite groups, Math. Z. 230 (1999) 509-527 MathReview

[7] B H Bowditch, Peripheral splittings of groups, Trans. Amer. Math. Soc. 353 (2001) 4057-4082 MathReview

[8] I Bumagin, On definitions of relatively hyperbolic groups, Proc. Amer. Math. Soc. (to appear)

[9] J Cannon, The combinatorial structure of cocompact discrete hyperbolic groups, Geometriae Dedicata 16 (1984) 123-148 MathReview

[10] D Collins, C Miller, The conjugacy problem and subgroups of finite index, Proc. London Math. Soc. 34 (1977) 535-556 MathReview

[11] F Dahmani, Classifying space and boundary for relatively hyperbolic groups, Proc. London Math. Soc.(3) 86 (2003) 666-684 MathReview

[12] F Dahmani, Combination of convergence groups, Geom. Topol. 7 (2003) 933-963 MathReview

[13] M Dehn, Über unendliche diskontinuierliche Gruppen, Math. Ann. 71 (1912) 116-144

[14] B Farb, Relatively hyperbolic groups, Geom. Func. Anal. 8 (1998) 810-840 MathReview

[15] A Juhász, Extension of group presentations and relative small cancellation theory. I, Internat. J. Algebra Comput. 10 (2000) 375-398 MathReview

[16] E Ghys, P dela Harpe, Editors, Sur les groupes hyperboliques d'après Mikhael Gromov, Progress in Mathematics 83, Birkhauser (1990) MathReview

[17] B Goldfarb, Novikov conjectures and relative hyperbolicity, Math. Scand. 85 (1999) 169-183 MathReview

[18] M Gromov, Hyperbolic groups, from: "Essays in group theory", (S Gersten, editor) Math. Sci. Res. Inst. Publ. 8, Springer (1987) 75-263 MathReview 
[19] GC Hruska, Nonpositively curved 2-complexes with isolated flats, Geom. Topol. 8 (2004) 205-275 MathReview

[20] I Kapovich, P Schupp, Relative hyperbolicity and Artin groups, Geom. Dedicata (to appear)

[21] O G Kharlampovich, A G Myasnikov, Irreducible affine varieties over a free group II, J. of Algebra 200 (1998) 517-570 MathReview

[22] O G Kharlampovich, A G Myasnikov, V N Remeslennikov, D E Serbin, Subgroups of fully residually free groups: algorithmic problems, Contemp. Math. 360 (2004) 63-101

[23] I Lysënok, Some algorithmic properties of hyperbolic groups (Russian), Izv. Akad. Nauk SSSR Ser. Mat. 53 (1989) 814-832; translation in Math. USSR-Izv. 35 (1990) 145-163 MathReview

[24] H Masur, Y Minsky, Geometry of the complex of curves I: Hyperbolicity, Invent. Math. 138 (1999) 103-149 MathReview

[25] A Mostowski, On the decidability of some problems in special classes of groups, Fund. Math. 59 (1966) 123-135 MathReview

[26] A G Myasnikov, V N Remeslennikov, DE Serbin, Regular free length functions on Lyndon's free $\mathbb{Z}[t]$-group $F^{\mathbb{Z}[t]}$, Contemp. Math. (to appear)

[27] D Osin, Relatively hyperbolic groups: Intrinsic geometry, algebraic properties, and algorithmic problems, Mem. Amer. Math. Soc. (to appear)

[28] D Osin, Weak hyperbolicity and free constructions, Contemp. Math. (to appear)

[29] D Rebbechi, Algorithmic Properties of Relatively Hyperbolic Groups, Ph.D. thesis, Rutgers (2000)

[30] Z Sela, Diophantine geometry over groups I, Publ. Math. Inst. Hautes Études Sci. 93 (2001) 31-105 MathReview

[31] A Szczepanski, Relatively hyperbolic groups, Michigan Math. J. 45 (1998) 611618 MathReview

[32] A Szczepanski, Examples of relatively hyperbolic groups, Geom. Dedicata 93 (2002) 139-142 MathReview

[33] A Yaman, A topological characterization of relatively hyperbolic groups, J. Reine Angew. Math. 566 (2004) 41-89 MathReview

Department of Mathematics and Statistics, Carleton University

1125 Colonel By Drive, Herzberg Building

Ottawa, Ontario, Canada K1S 5B6

Email: bumagin@math.carleton.ca

Received: 5 May 2002 Revised: 2 July 2003 